\title{
The Effectiveness of Provider Initiated Testing and Counselling within Nutrition Clinics for Malnourished Children less than 5 years in Adamawa State, Nigeria
}

\author{
Article by Samson Olufemi Adeyemi ${ }^{1}$, K U Suru ${ }^{1}$, J E Chiegil ${ }^{1}$, O F Martins ${ }^{2}$ \\ 1, 2, ${ }^{3}$ Texila American University, Guyana \\ ${ }^{2}$ Department of Public Health, Federal Medical Centre Yola, Adamawa State, Nigeria \\ E-mail: sassin33@yahoo.com ${ }^{1}$
}

\begin{abstract}
Background: Paediatric HIV infection and malnutrition remain two major health issues in Nigeria. Malnutrition is one of the clinical presentation of HIV in children who survive infancy undiagnosed, diagnoses usually occurs on presentation to health care services. We investigated the effectiveness of integrating provider-initiated testing and counselling (PITC) with nutrition services for children less under 5 years attending primary care clinics.

Methods: After an evaluation of HTC services for malnourished children aged 6-59 months in 2 primary health care facilities offering community management of acute malnutrition (CMAM) sites in Adamawa State, Nigeria, PITC was integrated into nutrition services. The change in the proportion of eligible children offered and receiving HIV tests and yield of HIV-positive diagnosis were compared for periods of 6 months before the integration of PITC and 6 months after the integration of PITC into CMAM OTP.

Results: There were 440 and 278 malnourished children eligible for HIV testing before and after the integration of PITC within nutrition services. The proportion of eligible malnourished children offered testing increased from 59\% to $100 \%$ and test uptake improved from 50\% to $98 \%$ in PITC integration period compared with before PITC integration period. The yield of HIV diagnosis increased from 0 to $1.5 \%$, and a malnourished child attending the clinic post intervention had 0.03 increased adjusted risk (95\% CI: 0.01 to 0.08) of receiving an HIV test in PITC integration period compared with preintervention period.
\end{abstract}

Conclusion: PITC integration into CMAM intensified paediatric HIV case finding.

Keywords: Nigeria, HIV, Malnourished Children, PITC.

\section{Introduction}

HIV is a major health challenge and one of the leading causes of death from infectious disease worldwide. It was estimated in 2015 that about 36.7 million people were living with HIV and about 2.1 million new infections occurred globally, 150,000 of those among children (UNAIDS, 2016). SubSaharan Africa remained the most hit region with about 25.6 million people living with HIV, and twothirds of new HIV infections globally occurred in this region. Children are lagging behind in the global response to HIV, resulting in major gaps around case finding and early infant diagnosis (EID), access to life-saving treatment as well as prevention.

In 2015, only $49 \%$ of an estimated 1.8 million children living with HIV had access to the lifesaving medicines (UNAIDS, 2016). Even though there is an improvement in contrast to $21 \%$ in 2010, more than still needed to be done because half of the children in need of treatment do not have access. Nigeria has the second highest burden of HIV/AIDS globally with an estimated 3.0 million people living with HIV (PLHIV) and 190,950 new infections in 2015. About 260,000 children aged 0-14 years were living with HIV in Nigeria in 2015 (UNAIDS, 2016), 41,000 new infections occurred among children, and only $17 \%$ of children living with HIV had access to antiretroviral therapy (UNAIDS, 2016). Paediatric HIV treatment has consistently lagged behind adult treatment.

HIV testing for HIV-exposed infants is available as part of the prevention of mother-to-child transmission (PMTCT) programs. However, in the absence of PMTCT services, between 30 and $45 \%$ of infants of HIV positive mothers will be infected with HIV. Many HIV-exposed children do not pass 
through the PMTCT program and there are high rates of loss to follow-up (LTFU) along the PMTCT cascade. National program data for the year 2014 show that 63,350 HIV-positive pregnant women received antiretroviral drugs to reduce the risk of mother-to-child transmission during pregnancy and delivery. In contrast, only 8802 infants received an HIV test within two months of birth (Federal Ministry of Health, 2015). The diagnosis of HIV for most children, who survive infancy untreated, depends largely on testing in the health care services when they present with clinical manifestations of HIV-indicators such as malnutrition (Shroufi et al. 2013, Lowenthal et al. 2014). Those who commenced HIV treatment at immune-compromised stage have worse treatment outcomes (Palella et al. 2003).

According UNICEF, as at 2013, about 37\% of children, or 6 million children under 5 years in Nigeria are chronically malnourished or low height for age, over half of them severely. In addition, $18 \%$ of children suffer from wasting (acutely malnourished), half of them severely and $29 \%$ of children are underweight (both acutely and chronically malnourished (UNICEF, 2014). There is disparity in prevalence of malnourished children in the different geopolitical regions in Nigeria. Children from the North-West and North-East geopolitical regions are more vulnerable to malnutrition than children from other geopolitical regions (UNICEF, 2014).

Adamawa is located in the North-Eastern region of Nigeria, with an estimated population of 4 million. The state had witnessed fluctuations in the pattern of the ANC HIV sentinel prevalence between 1999 and 2010 showing prevalence of $4.5 \%$ in 2001 to $7.5 \%$ in 2003, declining to $4.2 \%$ in 2005 and rising to $6.8 \%$ in 2008 but declined again to $3.8 \%$ in 2010. (ADSACA, 2016). In 2015, prevalence of HIV in Adamawa State was 2.5\% with an estimated 50,433 People Living with HIV (PLHIV), adult ART achievement of 25,341 (50.2\%), and pediatric ART achievement of 1,252 (25.4\%) out of estimated 4,935 children 0-14 years living with HIV (Federal Ministry of Health, 2016). Pediatric HIV treatment is lagging behind adult in Adamawa state. One of the challenges affecting pediatric HIV treatment is low case find in children. In other to close this gap there is the need to intensify pediatric HIV case finding mostly in area with low prevalence. Adamawa state HIV prevalence is below the national value $(3.0 \%)$, and it is one of the states in Nigeria with high prevalence of malnourished children under 5 years. This prompted the need for integration of HIV and nutrition services as a strategy to intensify pediatric HIV case finding.

HIV testing is the entry point to access HIV care. Client-initiated testing (CITC) approach also known as voluntary counselling and testing were used in the early stages of the HIV epidemic. The number of people that avail themselves for CITC was very low due to the fear of stigma and discrimination, thereby increasing missed opportunities for prevention. This lead to the integration of routine HIV testing into provider-initiated testing and counselling (PITC) in health facilities by WHO/UNAIDS in 2007 in order to re-conceptualize CITC and increase earlier HIV diagnosis and access to related services (WHO/UNAIDS, 2007). PITC is increasingly being promoted in clinical settings in countries with high HIV burden, a system whereby health care providers are regularly initiating an offer of HIV testing, regardless of the reason for presentation, in a context in which there is an assured provision of, or referral to, good preventive and treatment (Branson et al. 2006; UNAIDS 2004).

Although the WHO recommends PITC for all areas with generalized epidemics (WHO, 2007), CITC model is predominantly being practiced for HIV testing in many low and middle income countries (Matovu \& Makumbi, 2007). Though the goal of PITC is to deliver HIV testing to a high number of health service users, coverage remains inadequate, especially among children. This strategy depends on both the health care provider taking the lead to offer testing and the client agreeing to test. In practice, the proportion of individuals offered HIV testing differs, and is influenced by provider-perceived risks and benefits of testing the relationship between provider and client, client demographics, and logistical constraints (Roura et al. 2013).

In order to reduce the influence of health care workers on testing yield, PITC is being delivered as part of integrated services. PITC was integrated into specialist services such as PMTCT program, tuberculosis, and sexually transmitted disease services, to be conducted routinely as part of each clinical consultation and must be refused by the client if not desired, rather than individual HCW choosing whether or not to test on a case-by-case basis (Leon et al. 2010). In children, PITC integration with 
specialist services has been shown to be acceptable, feasible and effective within paediatric inpatient wards and immunization services (Rollins et al. 2009; 2011; Kankasa et al. 2009), also in sexually transmitted infections and TB services (Leon et al. 2010; Pope et al. 2008; CDC 2008). For years optout antenatal HIV testing and counselling has successfully increased HIV testing as a standard part of prevention of mother to child transmission (PMTCT) programs (CDC 2004; Moses et al. 2008; Chandisarewa et al. 2007). Lack of confidence in counselling children, and inadequate knowledge of the burden of HIV in children are among the major barriers to the delivery of PITC to children by HCW.

In 2015, Adamawa State Primary Health Care Development Agency in collaboration with UNICEF, integrated HIV testing services with nutrition intervention, for malnourished children under 5 years, using PITC approach, to intensify pediatric HIV case finding and linkage to treatment. This strategy is currently being implemented in 90 PHCs (CMAM sites). However, no study has been conducted on the effectiveness of this strategy of integrating PITC into nutrition services in Nigeria. Hence, the need for this research which aimed to explore the effectiveness of integrating PITC into nutrition services for malnourished children less than 5 years in Adamawa State, Nigeria. The effectiveness of the strategy was defined in terms of proportion of children offered and receiving HIV test, and the yield of HIV test. This study was limited by the sample size before and after the intervention, and our inability to explore the reasons for HIV test refusal.

\section{Methods}

Prior to the integration of PITC in CMAM, two of the sites were offering HIV testing services as part of services rendered to patients. Only these two sites met the criteria for inclusion in this study. Purposive sampling (Neyman, 1934) was used for sites selection. Malnourished children under 5 years accessing CMAM services in these two nutrition clinics were evaluated for the period of 12 months. Malnourished children who received accessed CMAM before the integration of PITC into nutrition services were evaluated retrospectively for six months as control, and a prospective evaluation of malnourished children who accessed CMAM after the integration of PITC into nutrition services were used as for the experiment.

A six-month evaluation of HIV testing services for malnourished children less than 5 years was conducted in 2 primary health care clinics (PHCs) in high density wards in Adamawa State, Nigeria, between early April and late September 2015. One of the health facilities Demsa PHC, is located in Demsa ward of Demsa local government area, and the other Ajiya Health Clinic Jimeta, is located in Ajiya ward of Yola North local government area. Each clinic serves 1 ward and provides comprehensive outpatient primary care, including maternal and child health services, acute care, HIV testing services, PMTCT, antenatal, delivery, and postnatal services. The two health facilities are also nutrition clinics delivering community management of acute malnutrition out patient therapeutic programme (CMAM OTP). Clinical services are delivered by community health extension workers (CHEWS). HIV counselling and testing services commenced in one of the facilities since 2013 and the other around March 2015, and it is based on the National Guidelines. HIV testing in PHCs is usually performed by trained counsellors (health care worker) who have undergone certified training in HIV counselling and testing.

Activities were initiated in mid-September 2015 in preparation for the integration of PITC into nutrition services (CMAM). These activities were supported by UNICEF and Adamawa State Primary Health Care Development Agency. A 5-day residential training course was conducted for the CHEWS, who are responsible for delivery CMAM services to malnourished children. The training focused mainly on integration of PITC testing model with CMAM, including issues relating to testing children, the burden of HIV among children, counselling of caregiver in CMAM, framework for consent and guardianship, PITC in CMAM flow chart, benefits of testing malnourished children and the benefits of early linkage to treatment. They were also trained extensively on how to implement opt-out testing strategy and documentation of services into the data tools. A mentoring program in integrated paediatric HIV and nutrition was formed to provide HCW with ongoing support. Buffer supply of HIV testing kits was made available to ensure an uninterrupted supply. The outcome of PITC integration with CMAM was evaluated for six-months period of implementation from early October 2015 to late March 2016. 
PITC was offered routinely as part of general health education to all caregivers who present to the health facility with their children aged 6-59 months for CMAM, in form of group counselling (Fig. 1). The nutrition status of all the children are evaluated through mid-upper arm circumference (MUAC) assessment using MUAC strip. Verbal consent to proceed with HIV test is obtained from the mother or caregiver of a child who is red on MUAC (malnourished). HIV testing was conducted unless the caregiver specifically declined permission, as per national guidelines. The standard HIV testing algorithm recommended by the national guideline was used; a rapid HIV antibody testing kit (Determine) was used with all positive tests confirmed by a second rapid antibody test (Uni-Gold). A discrepant test result was resolved using a third tie-breaker test (Stat-Pak). Malnourished children who less than 18 months who reacted to rapid antibody test were referred for early infant diagnosis (EID) using DNA-PCR testing according to the national guidelines. The mother of a malnourished child who tested positive or reacted to the antibody test was offered HIV test also. All malnourished children including those tested for HIV, regardless of the test result, and those who caregiver declined testing are assessed for appetite testing and admitted into the CMAM OTP in line with the national guideline for CMAM. Malnourished children admitted into CMAM OTP are given ready to use therapeutic food (RUTF) and exit from the health facility. The children are to revisit once in a week on CMAM day for refill of RUTF for a period of 8 weeks when their nutrition status will be re-evaluated based on the outcome of the CMAM treatment received. PITC is offered routinely to all caregivers with their children on CMAM days including those who declined testing during the first visit. Ethical approval for the study was obtained from the Adamawa State Ministry of Health, department of planning and statistics.

Data on socio-demographics of the children and caregiver, number of attendances of malnourished children aged 6-59 months and the number of tests offered and accepted were collected prospectively for 6 months during the implementation of PITC integration in CMAM OTP. The same data of HIV testing for malnourished children of this age group admitted into CMAM OTP during the period of 6month before the implementation of PITC in CMAM integration were also collected. The data were analysed using based on percentages and relative risk calculated according (Altman 1991) with online free statistical calculators (Medcalc, 2017). The proportion of malnourished children being offered and accepting testing, the yield of HIV-positive diagnosis (defined as the number of children testing positive among all children eligible for testing), and reasons for not being tested for HIV were compared before and after the integration of PITC in CMAM OTP. The proportion not tested due to a specific reason were calculated and stratified by 6 months before PITC integration with CMAM and 6 month PITC integration with CMAM period using the total number of children eligible during a period as a denominator. Modified Poisson regression was used to calculate the risk of being tested before and after the intervention.

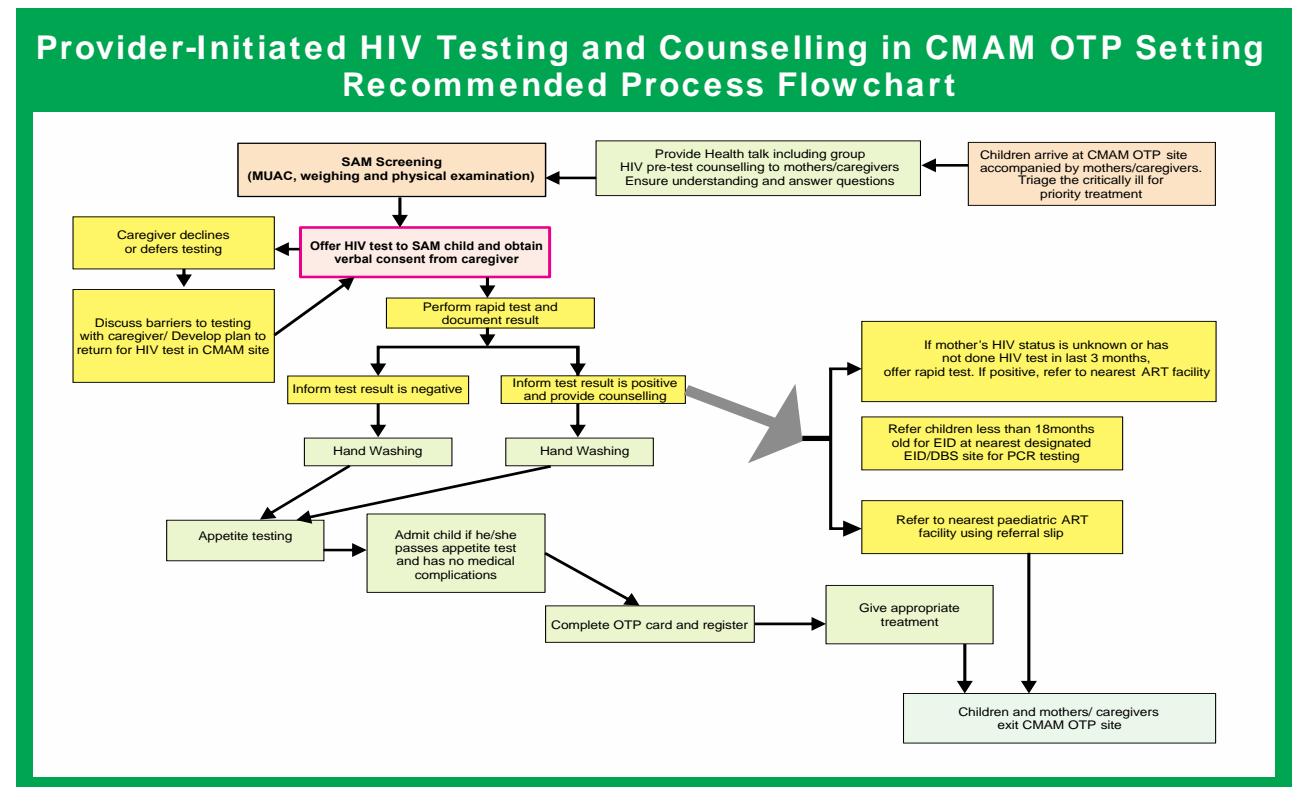

Figure 1. PITC in CMAM flowchart 


\section{Results}

A total of 440 and 278 malnourished children were eligible for HIV testing in the period before PITC integration and period after PITC integration respectively (Fig. 2). The proportion of eligible severe acute malnourished (SAM) children offered testing increased from 59\% to $100 \%$ after the integration of PITC into CMAM OTP (Table 1). Half of the malnourished children were not offered HIV test. A total of 221(50\%) malnourished children out of the 440 who accessed nutrition services in preintervention period were not offered HIV test. In contrast, all the malnourished children who accessed nutrition services during the intervention period were offered HIV test.

Test uptake among children offered a test improved from $85 \%$ to $98 \%$ during the period of PITC integration into CMAM OTP. The percentage of children testing HIV positive among all SAM children testing (yield) increased from $0 \%$ to $1.4 \%$, comparing the period before and after integration integration of PITC into CMAM OTP. The improvement in the number of test offered and accepted continued throughout the 6 months of PITC integration into CMAM site (Table 1).

The proportion of SAM children attending CMAM at PHCs, who did not receive a test, fell from $50 \%$ with PITC not integrated into CMAM to $1.8 \%$ with PITC integrated into CMAM site. A SAM child attending primary health care clinics post intervention period has increased chance (adjusted risk ratio 0.03 ; $95 \%$ CI: 0.01 to 0.08 ) to receive an HIV test compared with the pre-intervention period (Fig. 3)

Though we did not find out the reasons for HIV test refusal, a total of 39 (15\%) of the 260 malnourished children under 5 years (caregivers) offered HIV test declined testing in pre-intervention period. In comparison, only 5 (1.8\%) of the 278 malnourished children offered HIV test declined testing in the period of PITC integration into nutrition services.
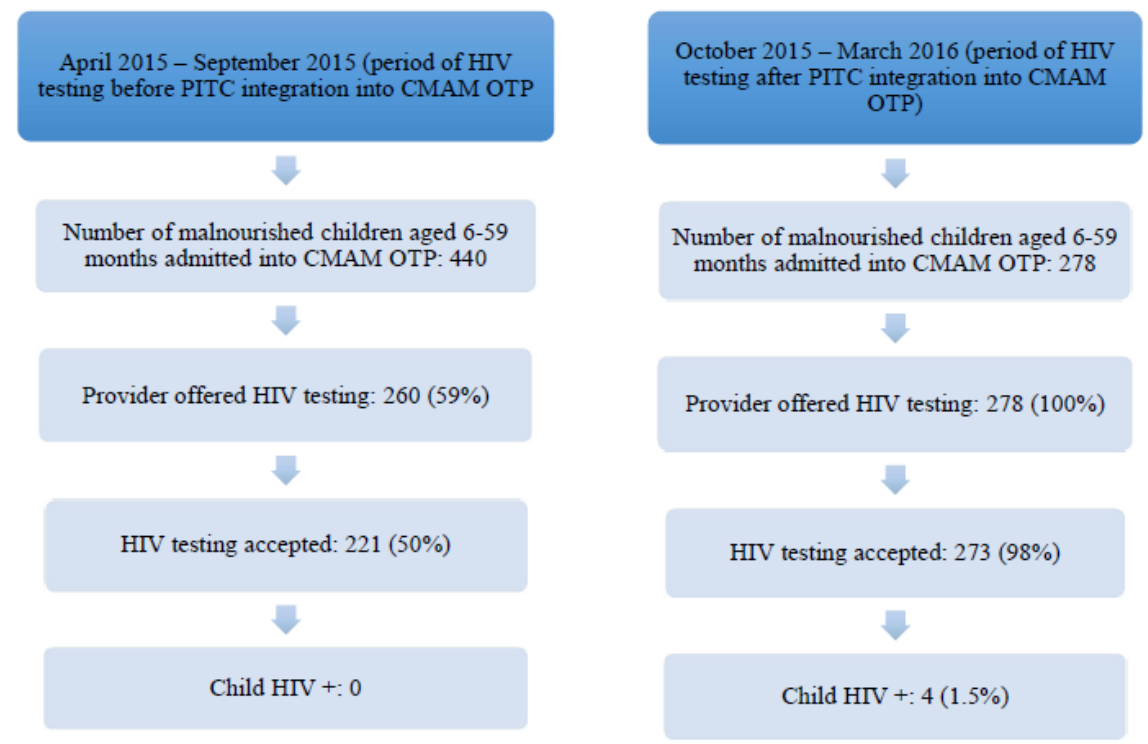

Figure 2. Number of malnourished children aged 6-59 months who offered testing, underwent testing, and tested HIV-positive during periods before and after PITC integration into CMAM OTP respectively 
DOI: $10.21522 /$ TIJPH.2013.05.04.Art021

ISSN: $2520-3134$

Table 1. Number of malnourished children offered Testing, Accepting Testing and Testing Positive per Month

\begin{tabular}{|c|c|c|c|c|c|c|}
\hline Period & $\begin{array}{l}\text { Number of } \\
\text { Children } \\
\text { Eligible } \\
\text { for } \\
\text { CMAM }\end{array}$ & $\begin{array}{l}\text { Provider } \\
\text { Offered } \\
\text { HIV } \\
\text { Testing, N } \\
(\%)\end{array}$ & $\begin{array}{l}\text { HIV } \\
\text { Testing } \\
\text { Accepted, } \\
\text { N }(\%)\end{array}$ & $\begin{array}{l}\text { \% of } \\
\text { Children } \\
\text { Tested for } \\
\text { HIV } \\
\text { Among All } \\
\text { Eligible }\end{array}$ & $\begin{array}{l}\text { Children } \\
\text { Testing } \\
\text { HIV +, } \\
\text { N }(\%)\end{array}$ & $\begin{array}{l}\text { \% of } \\
\text { Children } \\
\text { Testing } \\
\text { HIV + } \\
\text { Among All } \\
\text { Eligible }\end{array}$ \\
\hline April, 2015 & 83 & $60(75)$ & $51(85)$ & 61 & $0(0)$ & 0 \\
\hline May, 2015 & 83 & $55(66)$ & 49 (89) & 59 & $0(0)$ & 0 \\
\hline June, 2015 & 70 & $40(57)$ & $28(70)$ & 40 & $0(0)$ & 0 \\
\hline July, 2015 & 99 & $60(60)$ & $52(86)$ & 52 & $0(0)$ & 0 \\
\hline $\begin{array}{l}\text { August, } \\
2015\end{array}$ & 55 & $23(41)$ & $21(91)$ & 38 & $0(0)$ & 0 \\
\hline $\begin{array}{l}\text { September, } \\
2015\end{array}$ & 50 & $22(44)$ & $20(90)$ & 40 & $0(0)$ & 0 \\
\hline $\begin{array}{l}\text { Total } \\
\text { before } \\
\text { PITC } \\
\text { integration } \\
\text { into } \\
\text { CMAM } \\
\text { OTP }\end{array}$ & 440 & $260(59)$ & $221(85)$ & 50 & 0 (0) & (0) \\
\hline $\begin{array}{l}\text { October, } \\
2015\end{array}$ & 66 & $66(100)$ & $66(100)$ & 100 & $2(3.0)$ & 3.0 \\
\hline $\begin{array}{l}\text { November, } \\
2015\end{array}$ & 35 & $35(100)$ & $35(100)$ & 100 & $1(2.8)$ & 2.8 \\
\hline $\begin{array}{l}\text { December, } \\
2015\end{array}$ & 41 & $41(100)$ & $40(97)$ & 97 & $0(0)$ & 0 \\
\hline $\begin{array}{l}\text { January, } \\
2016\end{array}$ & 60 & $60(100)$ & $60(100)$ & 100 & $0(0)$ & 0 \\
\hline $\begin{array}{l}\text { February, } \\
2016\end{array}$ & 37 & $37(100)$ & $33(89)$ & 89 & $1(3.0)$ & 2.7 \\
\hline $\begin{array}{l}\text { March, } \\
2016\end{array}$ & 39 & $39(100)$ & 39 (100) & 100 & $0(0)$ & 0 \\
\hline $\begin{array}{l}\text { Total } \\
\text { After } \\
\text { PITC } \\
\text { integration } \\
\text { into } \\
\text { CMAM }\end{array}$ & 278 & $278(100)$ & $273(98)$ & 98 & $4(1.5)$ & 1.4 \\
\hline
\end{tabular}




\section{Free statistical calculators}

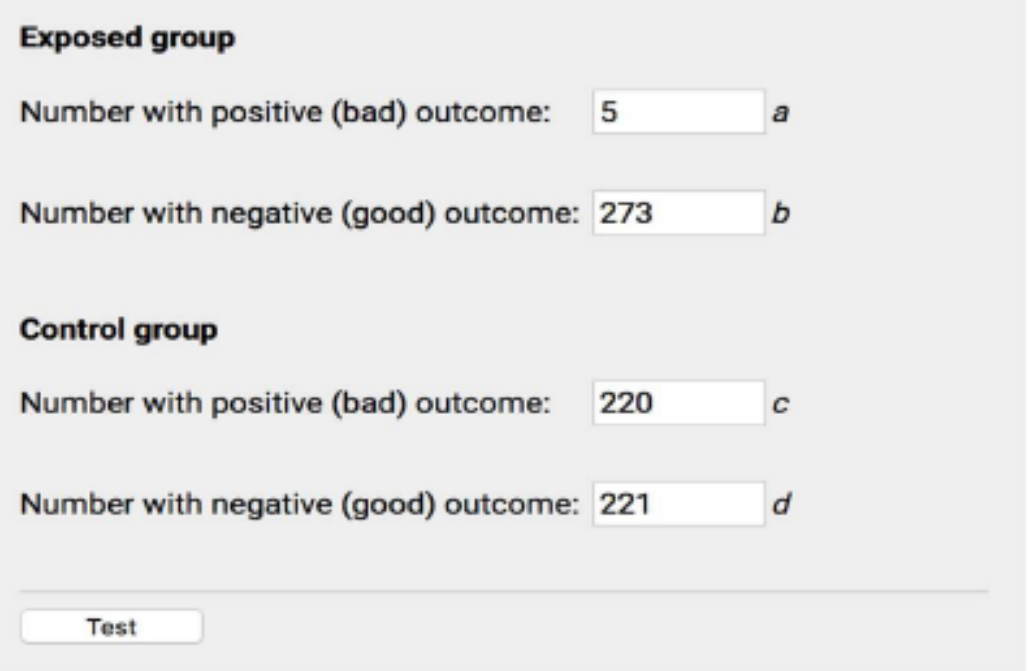

\section{Result}

\begin{tabular}{|l|l|}
\hline Relative risk & 0.0361 \\
$95 \% \mathrm{Cl}$ & 0.0150 to 0.0864 \\
\hline z statistic & 7.455 \\
\hline Significance level & $\mathrm{P}<0.0001$ \\
\hline NNT (Benefit) & 2.080 \\
\hline $95 \% \mathrm{Cl}$ & 1.849 (Benefit) to 2.376 (Benefit) \\
\hline
\end{tabular}

\section{Computational notes}

The relative risk (RR), its standard error and 95\% confidence interval are calculated according to Altman, 1991

The relative risk ratio is given by

$$
R R=\frac{a /(a+b)}{c /(c+d)}
$$

With the standard error of the log relative risk being

$$
\operatorname{SE}\{\ln (R R)\}=\sqrt{\frac{1}{a}+\frac{1}{c}-\frac{1}{a+b}-\frac{1}{c+d}}
$$

and $95 \%$ confidence interval

$$
95 \% \mathrm{CI}=\exp (\ln (R R)-1.96 \times \mathrm{SE}\{\ln (R R)\}) \quad \text { to } \quad \exp (\ln (R R)+1.96 \times \operatorname{SE}\{\ln (R R)\})
$$

Where zeros cause problems with computation of the relative risj or its standard error, 0.5 is added to all cells (a, b, c, d) (Pagano \& Gauvreau, 2000; Deeks \& Higgins, 2010).

\section{Number needed to treat (NNT)}

The number needed to treat (NNT) is the estimated number of patients who need to be treated with the new treatment rather than the standard treatment (or no treatment) for one additional patient to benefit (Altman 1998).

A negative number for the number needed to treat has been called the number needed to harm. 
MedCalc uses the terminology suggested by Altman (1998) with NNT (Benefit) and NNT (Harm) being the number of patients needed to be treated for one additional patient to benefit or to be harmed respectively.

The $95 \%$ confidence interval is calculated according to Daly (1998) and is reported as suggested by Altman (1998).

Increased adjusted risk of receiving an HIV test in PITC integration period compared with preintervention period.

\section{Discussion}

To our knowledge, this is the first study in Adamawa, Nigeria setting to compare HIV testing acceptance, and prevalence rates based upon screening protocol-PITC opt-out testing integration into nutrition clinic and PITC testing per client based on clinical presentation to primary health care clinics. We found HIV testing offered, accepted and yield to be comparable between the two groups. Result from this study shows that PITC integration into CMAM OTP, with adequate training and mentoring of $\mathrm{HCW}$, can increase the yield of HIV test for malnourished children attending primary health care clinics. The results were sustained over the period of 6 months.

The findings from this study shows that delivering PITC on case-by-case basis (Leon et al. 2010) can reduce the yield of HIV testing for SAM children attending primary health care clinics for nutrition intervention. The main reason that HIV testing did not occur attributed to HCW factors. Most of the health workers complained of not been able to offer HIV test case-by-case due to the effect of workload, attending to large number of SAM children on CMAM days, including new and revisit clients. This occur during the period before the PITC integration into CMAM. Malnourished children who presented to the health facility were not offered HIV test by the health worker, even with the clinical condition that could suggest the strong need for HIV test. However, because the health workers have to offer test to individual malnourished child, and considering the large number of children who present, only a few of them were offered HIV test by the health workers. In addition, despite the recommendation of WHO that PITC should be offered to all clients (WHO, 2007) this study shows that only 221 of all the 440 malnourished children who attended the nutrition clinics before the integration of PITC received HIV testing. This further support the evidence that the standard of care testing and counselling programs in most parts of sub-Saharan Africa still selectively target individuals who either request testing or possess clinical indications for it (Matovu \& Makumbi, 2007). The decision to offer HIV test to SAM children depends on the HCWs' knowledge of perceived-risk assessment relationship with client because despite the SAM children presenting with clinical indications only 59\% were offered HIV testing.

However, PITC integration into CMAM OTP has shown to increase acceptance of HIV testing from $85 \%$ pre-intervention to $98 \%$ post intervention. The integration took care of the barrier of a malnourished child not been offered HIV test due to knowledge gap of the health care worker on perceived risk assessment. This could also be counted as one of the gains of integrating PITC into CMAM because it reduces the burden of offering HIV test on case-by-case basis. Integration of PITC into specialist services will help to increase not only the number of clients offered HIV testing, but also the acceptability of HIV testing (CDC 2004; Moses et al. 2008; Chandisarewa et al. 2007). The number of caregivers who opt out after HIV test was offered decreased during the period of intervention when compared with period before intervention. About $17 \%$ of caregivers opt-out before the integration and $1.7 \%$ of caregivers opt out during the period of PITC integration into CMAM OTP. The reasons why care givers declined were not documented in this study. This increase in number of clients who accepted the test could also be attributed to the training. The training received by the health care workers before the integration of PITC into CMAM increased their counselling skills, which in turn decrease the number of clients who opted out during the period of PITC integration into CMAM.

The yield of HIV testing is higher among SAM children children attending primary health care clinics for nutrition intervention when PITC is integrated into CMAM OTP. The low yield recorded during period before the integration could have resulted from increase number of malnourished children who were not offered HIV test due to health worker factor. This increase the missed opportunity of diagnosing a HIV positive child. Increase in number of clients who opted out also increased the missed opportunity of diagnosing a child to be HIV positive. However, these barriers to missed opportunity 
were reduced during the period of PITC integration into CMAM. A total of 4 malnourished children were diagnosed HIV positive out of the 273 malnourished children tested. The HIV prevalence among SAM children for this study is lesser than the HIV prevalence of Adamawa state (ADSACA, 2016). $1.5 \%$ of SAM children tested HIV positive during the intervention period. Other studies have also shown evidence of how integration of PITC into infant and paediatric services can increase the yield of HIV testing (Rollins et al. 2009; Kankasa et al. 2009). The result of this study will inform future policy development on integrated service delivery.

\section{Conclusion}

Although we observed low levels of test refusal during the period of intervention, one limitation of this study was our inability to explore the reasons for the refusal. The sample size before and after the intervention was is another limitation. PITC integration with nutrition services increased the proportion of malnourished children undergoing HIV testing, resulting in an overall increased yield of positive diagnosis. The chance of a malnourished child receiving HIV test in nutrition clinic increased with the integration of PITC into CMAM. PITC integration within nutrition services provides an effective approach to reduce missed HIV diagnosis in this age group. The integration of PITC into nutrition services is effective in intensifying paediatric HIV case finding. However, future research need to focus on linkage of HIV positive SAM children to ART treatment and care.

\section{Acknowledgments}

We thank the dedicated staff of Demsa PHC and Ajiya Clinic Jimeta in Adamawa state, Nigeria. We also thank the Adamawa State Ministry of Health, Adamawa State Primary Health Care development Agency, and UNICEF Nigeria for their assistance in supplying HIV test materials and identifying local ART referral sites.

\section{References}

[1]. ADSACA. (2016). Adamawa State Agency for the Control of HIV/AIDS: 2015 Annual Report. Yola: Adamawa State Agency for the Control of HIV/AIDS.

[2]. Branson, B., Handsfield, H., Lampe, M., \& al., e. (2006). Revised recommendations for HIV testing of adults, adolescents, and pregnant women in health-care settings. MMWR Recomm Rep, 55, 1-17.

[3]. Chandisarewa, W., Stranix-Chibanda, L., \& Chirapa, E. e. (2007). Routine offer of antenatal HIV testing ("opt-out" approach) to prevent mother-to-child transmission of HIV in urban Zimbabwe. Bull World Health Organ, 85, 843-850.

[4]. Federal Ministry of Health. (2015). GARPR. Abuja: NACA.

[5]. Federal Ministry of Health. (2016). National AIDS and STIs Control Programme: 2015 Annual Report on HIV/AIDS Health Sector Response in Nigeria. Abuja: Federal Ministry of Health.

[6]. Kankasa, C., Carter, R., Briggs, N., \& al, e. (2009). Routine offering of HIV testing to hospitalized pediatric patients at university teaching hospital, Lusaka, Zambia: acceptability and feasibility. J Acquir Immune Defic Syndr, 51, 202-208.

[7]. Leon, N., Naidoo, P., Mathews, C., \& al, e. (2010). The impact of provider-initiated (opt-out) HIV testing and counseling of patients with sexually transmitted infection in Cape Town, South Africa: a controlled trial. Implement Sci, 5, 8 .

[8]. Lowenthal, E., Bakeera-Kitaka, S., Marukutira, T., \& al, e. (2014). Perinatally acquired HIV infection in adolescents from sub-Saharan Africa: a review of emerging challenges. 14, 627-639.

[9]. Matovu, J., \& Makumbi, F. (2007). Expanding access to voluntary HIV counselling and testing in subSaharan Africa: alternative approaches for improving uptake, 2001-2007. Trop Med Int Health, 12, 1315-1322.

[10]. Medcalc. (2017). Free statistical calculators. Retrieved 2017, from MEDCALC easy-to-use statistical software: https://www.medcalc.org/calc/relative_risk.php.

[11]. Moses, A., Zimba, C., Kamanga, E., \& al., e. (2008). Prevention of mother-to-child transmission: program changes and the effect on uptake of the HIVNET 012 regimen in Malawi. AIDS, 22, 83-87.

[12]. Neyman, J. (1934). On the Two Different Aspects of the Representative Method: The Method of Stratified Sampling and the Method of Purposive Selection. Journal of the Royal Statistical Society, 97 (4), 558-625. 
DOI: $10.21522 /$ TIJPH.2013.05.04.Art021

ISSN: $2520-3134$

[13]. Palella, F. J., Deloria-Knoll, M., Chmiel, J., \& al, e. (2003). Survival benefit of initiating antiretroviral therapy in HIV-infected persons in different CD4+ cell strata. Ann Intern Med, 138, 620-626.

[14]. Pope, D., Deluca, A., Kali, P., \& al, e. (2008). A cluster-randomized trial of provider-initiated (opt-out) HIV counseling and testing of tuberculosis patients in South Africa. J Acquir. Immune Defic Syndr, 48, 190-195.

[15]. Rollins, N., Mzolo, S., Moodley, T., \& al, e. (2009). Universal HIV testing of infants at immunization clinics: an acceptable and feasible approach for early infant diagnosis in high HIV prevalence settings. AIDS, 23, 18511857.

[16]. Roura, M., Watson-Jones, D., Kahawita, T., \& al, e. (2013). Provider-initiated testing and counselling programmes in sub-Saharan Africa: a systematic review of their operational implementation. AIDS, 27, 617-626. [17]. Shroufi, A., Gunguwo, H., Dixon, M., \& al., e. (2013). HIV-infected adolescents in southern Africa can achieve good treatment outcomes: results from a retrospective cohort study. AIDS 27, 1971-1978.

[18]. UNAIDS. (2016). Children and HIV Fact Sheet. Geneva: UNAIDS.

[19]. UNAIDS. (2016). HIV and AIDS estimates (2015). (UNAIDS, Producer) Retrieved August 20, 2017, from UNAIDS: http://www.unaids.org/en/regionscountries/countries/nigeria

[20]. UNAIDS. (2016). Nigeria -UNAids. Retrieved August 20, 2017, from UNAIDS 2016 estimates, unless otherwise noted: http://www.unaids.org/sites/default/files/media/documents/UNAIDS_GlobalplanCountryfactsheet_nigeria_en.p df

[21]. UNAIDS/WHO. (2004). UNAIDS/WHO Policy Statement on HIV Testing. Geneva: UNAIDS Global Reference Group on HIV/AIDS and Human Rights.

[22]. UNICEF. (2014). Malnutrition rates in children under 5 years. Retrieved 09 17, 2017, from Nutrition: https://www.unicef.org/nigeria/factsheets_NUTRITION_low.pdf.

[23]. US Centers for Disease Control and Prevention. (2004). Introduction of routine HIV testing in prenatal care-Botswana. MMWR Morb Mortal Wkly Rep, 53, 1083-1086.

[24]. US Centers for Disease Control and Prevention. (2008). Provider-initiated HIV testing and counseling of TB patients--Livingstone District, Zambia, September 2004-December 2006. MMWR Morb Mortal Wkly Rep, 57, 285-289.

[25]. WHO. (2007). World Health Organization (WHO) Guidance on provider-initiated HIV testing and counselling in health facilities. Retrieved from http://whqlibdoc.who.int/publications/2007/9789241595568_eng.pdf.

[26]. WHO/UNAIDS. (2007). Guidance on Provider-initiated HIV Testing and Counselling in Health Facilities, May 2007. Geneva: WHO. 